\title{
REVISIONES
}

\section{Aprendizaje de la escritura en las nuevas bases curriculares de Lenguaje y Comunicación: Nociones teóricas y modelos de escritura que subyacen a la propuesta curricular}

\author{
Learning to write in the new framework of Language and Communication: \\ Theoretical concepts and models underlying the curriculum

\section{Aprendizagem da escrita nas novas diretrizes curriculares de Linguagem e Comunicação: Conceitos e modelos teóricos da escrita subjacentes à proposta curricular}

\author{
Espinosa, María Jesús ${ }^{a}$; Concha, Soledad ${ }^{b}$ \\ ${ }^{a}$ Universidad Diego Portales - Universidad Alberto Hurtado, \\ Santiago, Chile. Teléfono: 984396500. \\ Correo electrónico: mariajesusea@gmail.com \\ ${ }^{\text {b} F a c u l t a d ~ d e ~ E d u c a c i o ́ n, ~ U n i v e r s i d a d ~ D i e g o ~ P o r t a l e s, ~ S a n t i a g o, ~ C h i l e . ~ T e l e ́ f o n o: ~ 56-2-6768503 . ~}$ \\ Correo electrónico: soledad.concha@mail.udp.cl
}

\begin{abstract}
RESUMEN
En este trabajo se analizan las bases curriculares 2012 de Lenguaje y Comunicación, específicamente en el ámbito de la enseñanza de la escritura de $1^{\circ}$ a $6^{\circ}$ básico, a la luz de los conceptos de metacognición y conciencia metalingüística, y de cinco modelos del proceso de escritura: el de Flower \& Hayes (1981), "decir el conocimiento" y "transformar el conocimiento" de Bereiter \& Scardamalia (1987, 1992), Hayes (2000), y el modelo del grupo DIDACTEXT (2003). El propósito de este análisis es identificar y describir las teorías que subyacen a las bases curriculares en el ámbito de la escritura. Asimismo, se busca determinar los énfasis, ambigüedades y vacíos detrás del currículum, en el marco de los conocimientos actuales sobre escritura.
\end{abstract}

Palabras clave: bases curriculares, escritura, metacognición, conciencia metalingüística, modelos de escritura.

\begin{abstract}
This article analyzes the requirements for writing, from grade 1 to 6 , as established in the 2012 Language and Communication Curriculum. The framework for this study involves the concepts of metacognition and metalinguistic awareness, and five models of the writing process: Flower \& Hayes (1981), the "knowledge telling" and "knowledge transformation" theory proposed by Bereiter \& Scardamalia (1987, 1992), Hayes (2000), and the DIDACTEXT model (2003). The aim of this study is, firstly, to identify, describe and explain the theories of writing that are at the core of this curriculum, in order to inform school teachers and university teachers of pedagogy. The second purpose of this study is to determine what is emphasized in the curriculum, as well as, what areas are ambiguous and what is left out of it, in the light of what we know today about the teaching of writing.
\end{abstract}

Key words: language curriculum, writing, metacognition, metalinguistic awareness, writing models.

\section{RESUMO}

Este trabalho analisa as bases curriculares de Linguagem e Comunicação 2012, especificamente no campo do ensino da escrita do grau 1 ao 6 , à luz dos conceitos de metacognição e consciência metalingüística, e cinco modelos do processo de escrita: Flower e Hayes (1981), "dizer o conhecimento" e "transformação do conhecimento" de Bereiter e Scardamalia (1987, 1992), Hayes (2000), e o modelo do grupo Didactext (2003). O objetivo desta análise é identificar e descrever as teorias subjacentes às diretrizes curriculares na área da escrita. $\mathrm{O}$ estudo também procura determinar a ênfase, ambigüidades e lacunas no interior da proposta curricular a fim de contribuir para a discussão sobre as exigências impostas aos nossos alunos no contexto do conhecimento atual sobre aprender a escrever.

Palavras chave: bases curriculares, escrita, metacognição, consciência metalingüística, modelos da escrita. 


\section{INTRODUCCIÓN}

Las Bases Curriculares de Lenguaje y Comunicación para la Enseñanza Básica, recientemente aprobadas por el Consejo Nacional de Educación, comenzaron a ser implementadas el 2012 y hoy ya se imparten hasta $6^{\circ}$ básico. Los cambios introducidos en este currículum de lenguaje suponen nuevos desafíos para los profesores y para las universidades e institutos que imparten la carrera de Pedagogía Básica, ya que establecen énfasis y exigencias en el aprendizaje del lenguaje, distintos a los de currículos anteriores, que los docentes -en ejercicio y en formación- deben conocer y manejar en aras de tomar las decisiones pedagógicas pertinentes para la enseñanza. Una de las principales diferencias que se observa respecto del currículo aprobado el 2009 es que las nuevas Bases Curriculares especifican con mayor detalle los aprendizajes que deben lograr los estudiantes en relación con la escritura, especialmente en relación con el proceso de escribir. Detrás de esta propuesta, que pone énfasis en el proceso de escritura como parte esencial del aprendizaje de esta habilidad, subyacen nociones teóricas que informan las decisiones didácticas al momento de la enseñanza. De acuerdo con esto, este trabajo se propone abordar las siguientes preguntas: ¿Qué nociones teóricas y modelos sobre el proceso de escritura hay detrás de los objetivos de aprendizaje de escritura de las Bases Curriculares? ¿Qué se dice en la literatura especializada sobre estas teorías? ¿Qué aspectos relevantes de estas nociones y modelos no son cubiertos por las Bases Curriculares de Lenguaje?

Considerando estas preguntas, el trabajo ha sido estructurado en dos partes. En la primera parte se describe lo que el currículum establece en torno al aprendizaje de la escritura y se analiza esta propuesta tomando en cuenta su finalidad y la manera en que se describen los objetivos. La segunda parte considera una revisión de la literatura sobre los principales modelos teóricos del proceso de escritura y sobre dos importantes nociones que subyacen a estos modelos: metacognición y conciencia metalingüística. Junto con esta revisión se incluye un análisis sobre los objetivos de aprendizaje de escritura, para determinar el grado de cercanía o lejanía entre ellos y la teoría examinada. El propósito de este análisis es, por una parte, identificar y describir las teorías que subyacen a las Bases Curriculares en el ámbito de la escritura, con el ánimo de informar a los docentes y directivos de las carreras de Pedagogía sobre aquellos aspectos del nuevo currículum que podrían suscitar una revisión de los cursos sobre didáctica de la escritura. Por otra parte, se busca identificar los énfasis, ambigüedades y vacíos detrás de la propuesta curricular del Ministerio de Educación, de manera de aportar información a la discusión sobre las exigencias que se plantean a nuestros alumnos, en el marco de los conocimientos actuales sobre el aprendizaje de la escritura.

\section{ESCRITURA EN LAS BASES CURRICULARES}

\subsection{FINALIDAD DE LOS OBJETIVOS DE APRENDIZAJE}

En la introducción de las Bases Curriculares, en el eje de escritura se declara que "la asignatura de Lenguaje y Comunicación busca que los estudiantes dominen las habilidades necesarias para expresarse eficazmente y usen la escritura como herramienta para 
aprender" (Ministerio de Educación, 2012a: 39). Es decir, hay un énfasis en la escritura como herramienta de comunicación y de aprendizaje. Desde la perspectiva de la escritura como forma de comunicación, se declara que un principio que debe guiar toda actividad de escritura es que siempre se escribe para alguien y para algo. Este principio se recoge, en un primer momento, en el objetivo referido a caligrafía, que está orientado a desarrollar conciencia del lector. Asimismo, en los objetivos referidos al proceso de escritura, también es posible identificar, aunque no tan claramente como en el de caligrafía, el principio de la escritura como comunicación, en cuanto prescriben la necesidad de que los alumnos planifiquen considerando su propósito y destinatario y revisen sus textos para transmitir sus ideas con claridad. Los objetivos de escritura de artículos informativos y otros textos no literarios también recogen el enfoque de escritura como herramienta de comunicación, ya que establecen que los estudiantes deben escribir estos textos "para comunicar información sobre un tema" y para "compartir impresiones sobre sus lecturas".

En cuanto al enfoque de la escritura como herramienta de aprendizaje, si bien está explicado en la introducción, no queda tan clara la incorporación de esta dimensión en los objetivos, a excepción del de planificación, que incluye la generación de ideas a partir de conversaciones, lluvia de ideas e investigaciones. En efecto, los objetivos del eje de escritura consideran el aprendizaje de estructuras textuales, conceptos básicos de gramática, reglas de ortografía y procesos de planificación, revisión y edición de los textos; pero no explicitan respecto de qué ni cómo los estudiantes "reflexiona[n] sobre el contenido de lo que se va a comunicar" (Ministerio de Educación, 2012a: 40), que, de acuerdo con lo señalado en la introducción, permitiría al estudiante aprender mientras escribe.

El eje de escritura de las Bases Curriculares, de acuerdo con la tabla de progresión publicada por el Ministerio de Educación (2012b), está organizado en siete líneas que agrupan los objetivos de aprendizaje: escritura libre, escritura de narraciones, escritura de artículos informativos, escritura de otros textos no literarios, legibilidad, procesos de escritura y manejo de la lengua.

El objetivo de la línea de escritura libre, como se declara en la introducción de la asignatura,

promueve la experimentación con diversos formatos, estructuras, soportes y registros que los alumnos pueden elegir y utilizar de acuerdo con sus propósitos comunicativos (...) para que (...) adquieran flexibilidad, descubran un estilo personal y desarrollen el gusto y la necesidad de escribir con diversos propósitos (Ministerio de Educación, 2012a: 40).

Según lo que establece este objetivo de aprendizaje, que se mantiene desde $1^{\circ}$ hasta $6^{\circ}$ básico, los estudiantes deben escribir frecuentemente textos de diversos géneros para desarrollar su creatividad y expresar sus ideas, lo que va en concordancia con lo expresado en la introducción. En este sentido, el currículum deja entrever una preocupación por el desarrollo de la motivación y de la autonomía en torno al aprendizaje de la escritura.

Los objetivos que corresponden a las líneas de escritura de narraciones (desde $2^{\circ}$ hasta $6^{\circ}$ básico $^{1}$ ) y escritura de artículos informativos (desde $2^{\circ}$ hasta $6^{\circ}$ básico) describen en forma bastante detallada la estructura propia de estos textos, lo que es coherente con

En $1^{\circ}$ básico se espera que los estudiantes sean capaces de escribir oraciones completas. 
la aproximación de la escritura guiada, descrita en la introducción, según la cual "Esta aproximación permite que los alumnos se familiaricen con las ventajas y los desafíos que presenta la estructura de cada género" (Ministerio de Educación, 2012a: 40). Por su parte, la línea de escritura de otros textos no literarios agrupa dos tipos de objetivos. En $3^{\circ}$ y $4^{\circ}$ básico, los estudiantes deben escribir una variedad de textos -cartas, instrucciones, afiches, reportes de una experiencia- para cumplir distintos propósitos, poniendo especial atención en el formato y la claridad del mensaje. En $5^{\circ}$ y $6^{\circ}$ básico, asimismo, los objetivos establecen que los alumnos aprendan a escribir impresiones sobre sus lecturas, "desarrollando un tema relevante del texto leído y fundamentando sus comentarios con ejemplos". En síntesis, todos estos objetivos, aunque los de narraciones y artículos informativos con más detalle que los de la línea "escritura de otros textos no literarios", destacan la importancia de que los estudiantes se familiaricen y aprendan las estructuras de algunos géneros discursivos propios de la escuela y la vida cotidiana. Por lo tanto, detrás de esta propuesta, si bien no se declara explícitamente, se puede inferir cierta preocupación porque los estudiantes reflexionen sobre la lengua y desarrollen un grado de conciencia respecto de los componentes del lenguaje.

Esta preocupación se observa con mayor claridad en la línea de objetivos de manejo de la lengua, específicamente los de gramática, que apuntan a que los estudiantes comprendan la función de las unidades morfológicas de la lengua (artículos, sustantivos, pronombres, adjetivos, adverbios y verbos). Aun cuando no se menciona explícitamente en los objetivos el interés por que los estudiantes reflexionen sobre el lenguaje sí se expresa en la introducción, en que se declara que "el enfoque comunicativo que sustenta esta propuesta curricular considera que los conocimientos gramaticales son una herramienta para reflexionar sobre el lenguaje y sus posibilidades en contextos concretos de comunicación" (Ministerio de Educación, 2012a: 40).

En cuanto a los objetivos de la línea de legibilidad, que se mantienen desde $1^{\circ}$ hasta $4^{\circ}$ básico, el propósito que subyace a ellos es el desarrollo de una caligrafía legible, aunque ni en la introducción ni en los mismos objetivos se declara si se debe privilegiar la letra imprenta o la letra manuscrita, por lo que esta decisión queda al arbitrio de la escuela. Como ya se dijo, este objetivo supone que los estudiantes desarrollen conciencia de un lector, ya que la importancia que se le da a la caligrafía radica en que la claridad de la letra facilita la comprensión de la persona que leerá el texto. Por lo demás, el objetivo de ortografía, de la línea de manejo de la lengua, que incluye el aprendizaje de las reglas de ortografía literal, acentual y las principales normas de ortografía puntual, también busca que los estudiantes se preocupen de su ortografía para facilitar la tarea del lector:

Esta aproximación contribuye a que los alumnos desarrollen, por una parte, la noción de que para comunicar mejor un mensaje la ortografía correcta es indispensable y, por otra, el hábito de prestar atención a este aspecto y revisar los textos con el fin de facilitar su lectura y su comprensión (Ministerio de Educación, 2012a: 40).

Por último, la línea de procesos de escritura considera dos objetivos por curso, desde $2^{\circ}$ hasta $6^{\circ}$ básico. El primer objetivo corresponde al aprendizaje de la planificación y el segundo contempla el aprendizaje de la escritura, la revisión y la edición de los textos. En la introducción de las Bases Curriculares se afirma que: 
Un escritor pone en práctica acciones complejas que implican una reflexión constante durante todo el proceso de escritura. La perspectiva de la escritura como proceso permite al alumno establecer propósitos, profundizar las ideas, trabajar con otros, compartir sus creaciones y centrarse en diferentes tareas necesarias para la producción (Ministerio de Educación, 2012a: 40).

De esta afirmación se desprende que la escritura debe abordarse como una actividad compleja que supone la lectura y transformación del texto antes de ser publicado. Al incluir dos objetivos de aprendizaje que se hacen cargo de esta propuesta, se enfatiza que aprender a escribir supone dominar procesos intermedios sin los cuales el texto escrito no está en condiciones de ser publicado. Esto quiere decir, por lo tanto, que el profesor debe adecuar la enseñanza y la evaluación para asegurar que los estudiantes no solo tengan oportunidades para escribir, sino también para planificar, revisar y editar todos los elementos detallados en los objetivos del proceso de escritura: el propósito y el destinatario del texto, la generación y organización de las ideas, el vocabulario, el uso de conectores, la ortografía y la presentación.

En resumen, las Bases Curriculares aquí revisadas abordan el aprendizaje de la escritura por medio de distintos tipos de objetivos que enfatizan aspectos diferentes de la misma. El objetivo de escritura libre promueve el desarrollo de la creatividad, la autonomía (para tomar decisiones sobre temas y formatos) y la motivación por la escritura. Los objetivos de escritura de narraciones, artículos informativos y otros textos no literarios destacan la importancia de manejar las estructuras textuales de los distintos géneros discursivos. Los objetivos de gramática apuntan a adquirir conocimientos que permitan al estudiante reflexionar sobre el lenguaje. Los objetivos de ortografía y de legibilidad buscan desarrollar conciencia del lector. Los objetivos de proceso de escritura describen distintos aspectos en los que el niño debe fijar su atención para producir un texto coherente y, en concordancia, consideran el desarrollo de actividades necesarias para elaborar un texto que facilite la comprensión por parte del lector y, por lo tanto, la comunicación de ideas.

\subsection{ANÁLISIS DE LA REDACCIÓN DE LOS OBJETIVOS DE APRENDIZAJE}

Un primer elemento que llama la atención respecto de los objetivos hasta aquí reseñados es la asimetría en el nivel de detalle de los mismos. Algunos -como los objetivos de escritura de narraciones y artículos informativos, los referidos al proceso de escritura y el de ortografía- se presentan considerablemente desagregados y especifican diversos aspectos que deben tomarse en cuenta para que los estudiantes logren los aprendizajes prescritos. Se estima que los detalles señalados permitirían orientar en alguna medida las decisiones pedagógicas, en tanto describen no solo conductas finales (por ejemplo, "planificar sus textos"), sino también las acciones que se encaminan hacia ellas ("estableciendo propósitos y destinatario, generando ideas a partir de sus conocimientos, etcétera). Adicionalmente, las viñetas con detalles se gradúan de modo tal de orientar un progreso en los objetivos a lo largo de los años escolares. Por ejemplo, para aprender a escribir narraciones, se espera que en segundo básico logren estructurarlas en inicio desarrollo y desenlace; luego, en tercero básico, a esto se agrega que los estudiantes deben poner atención a la secuencia lógica y a los conectores que utilizan; en cuarto básico, además de lo anterior, se espera que enriquezcan el lenguaje y las descripciones de sus historias; en quinto básico deben ser capaces de introducir diálogos y, en sexto, se espera que aseguren la coherencia de las oraciones. 
Si bien es difícil aventurar cómo cada docente implementará esta propuesta curricular, es interesante relevar una cierta intencionalidad de apoyo pedagógico para la implementación en los objetivos analizados. Por ejemplo, los objetivos de proceso de escritura entregan algunas orientaciones sobre cómo guiar el aprendizaje cuando, respecto de la planificación, se establece que los estudiantes generen ideas "a partir de lluvia de ideas, conversaciones e investigaciones"; es decir, se detalla lo que se espera que los alumnos logren y, en términos generales, cómo proceder pedagógicamente para apoyarlos. En el objetivo de escritura, revisión y edición, en los cursos más pequeños los estudiantes mejoran sus textos "a partir de sugerencias de los pares y el docente", mientras que en $5^{\circ}$ y $6^{\circ}$ básico deben ser capaces de "relee[r] a medida que escriben", es decir, se orienta de manera general la manera de realizar el proceso de corrección. Los demás objetivos, sin embargo, son mucho más amplios y carecen de una descripción exhaustiva que pudiera orientar las decisiones pedagógicas que deben tomar los profesores. Es el caso de objetivos como el de vocabulario: "incorporar de manera pertinente en la escritura el vocabulario nuevo extraído de textos escuchados o leídos". Como se observa en el ejemplo, la expresión "de manera pertinente" corresponde a la dimensión de cómo lograr el aprendizaje señalado, pero no especifica conductas intermedias que pudieran promover dicho aprendizaje.

En efecto, el análisis de asimetrías en el nivel de desarrollo con que se presentan los objetivos remite a una discusión clave sobre la finalidad que tienen las Bases Curriculares: a saber, prescribir los aprendizajes que deben lograr los estudiantes al término del año escolar. Como se señala en la introducción general a todas las asignaturas:

Los Objetivos de Aprendizaje relacionan en forma más explícita las habilidades, los conocimientos y las actitudes y evidencian en forma clara y precisa cuál es el aprendizaje que el estudiante debe lograr. Se conforma así un currículum centrado en el aprendizaje, que declara explícitamente cuál es el foco del quehacer educativo (Ministerio de Educación, 2012a: 12).

La opción por centrar el currículum en el aprendizaje de los estudiantes (y no en lo que los docentes enseñan) tendría una triple ventaja: por una parte, permite poner a los alumnos en el centro de los procesos de enseñanza y aprendizaje, además, entrega una base similar a todos los estudiantes del país y, por último, asegura la libertad de educación, que descansa en el tiempo de libre disposición y en la libertad para escoger los métodos de enseñanza que la escuela o el profesor estimen convenientes, siempre que permitan a los alumnos alcanzar los aprendizajes prescritos. Sin embargo, las señaladas ventajas acarrean el inconveniente de que no se informa el currículum sobre las estrategias didácticas más apropiadas para desarrollar los aprendizajes propuestos, lo que deja en manos de la formación inicial y continua la actualización en aquellas propuestas didácticas sobre las que se ha construido el currículum. Considerando la posición mandante del currículum y los procedimientos de implementación utilizados tradicionalmente en nuestro país, este proceso de actualización ocurre necesariamente con posterioridad a la publicación e implementación de las Bases Curriculares, razón por la que se vuelve relevante una discusión sobre la posibilidad de que el mismo documento pudiese orientar la actualización didáctica.

$\mathrm{Al}$ respecto, los objetivos que especifican con más detalle lo que los estudiantes deben lograr y que, según el análisis precedente, permitirían desprender una didáctica o, cuando menos, orientaciones más claras sobre cómo guiar el aprendizaje, estarían ofreciendo al 
cuerpo docente información complementaria a aquella disponible en los espacios formativos. En efecto, se estima que los objetivos más desagregados tendrían el potencial de ofrecer una mejor visualización de la progresión año a año de los aprendizajes, así como información sobre qué y cómo deben aprender los estudiantes. Otro espacio en el que el currículum podría proporcionar este tipo de información es en la introducción, que en la actual propuesta es bastante general y poco informativa respecto de la didáctica que favorecería la implementación de los objetivos de aprendizaje.

También en el ámbito del tipo de información que se provee a los docentes, en algunos casos las nociones teóricas que subyacen a la propuesta son bastante explícitas y permiten identificar con relativa claridad los autores y aportes que informan este currículum. Las fuentes citadas y las explicaciones de la introducción son especialmente útiles para identificar las teorías que sostienen a los objetivos de aprendizaje, lo que se logra especialmente en los objetivos referidos al proceso de escritura. Sin embargo, en otros casos, como los objetivos de gramática y de escritura de otros textos no literarios, no resulta evidente cuáles son las teorías que están detrás. Aunque este currículum, en comparación con el anterior, explicita algunas de las teorías que sustentan los objetivos de aprendizaje del eje de escritura ${ }^{2}$, aún se hace necesario exponer con mayor claridad las nociones y modelos teóricos que informan su didáctica, de manera que los docentes y académicos de las carreras de pedagogía y de la formación en servicio tengan los antecedentes necesarios para evaluar la propuesta en su justa medida y para tomar decisiones curriculares y bibliográficas en el marco de la formación que ofrecen. La segunda parte de este trabajo pretende contribuir a este propósito.

\section{METACOGNICIÓN Y CONCIENCIA METALINGÜÍSTICA}

\subsection{METACOGNICIÓN}

Si bien el concepto de metacognición no aparece mencionado en ninguna parte de las Bases Curriculares de Lenguaje y Comunicación, algunos elementos destacados en la introducción y los objetivos de aprendizaje permiten afirmar que la metacognición es un requisito para desarrollar a cabalidad los aprendizajes de escritura. A continuación se profundiza en este concepto, para luego analizar cómo se encuentra abordado en el currículum de Lenguaje.

En el capítulo 12 del libro The nature of intelligence, publicado en 1976, John Flavell describe uno de los problemas por los que los niños fallan en resolver desafíos cognitivos, aun cuando realizan todos los procedimientos debidos. Para Flavell (1976), se trata de un problema de metacognición, que define como el conocimiento que el individuo tiene sobre sus procesos y productos cognitivos y sobre los aspectos que influyen en dicho proceso: el ambiente, los requerimientos de la tarea y sus habilidades para

El ajuste curricular 2009 no integraba la bibliografía al documento oficial. Los fundamentos teóricos se encontraban en un documento diferente titulado "Fundamentos del ajuste curricular en el sector de Lenguaje y Comunicación": http://ww2. educarchile.cl/UserFiles/P0001/File/ajuste_curricular/Fundamentos_Lenguaje.pdf. En este se hace mención, sobre todo, a las nociones teóricas que sostienen el enfoque comunicativo de la propuesta, y se explican algunas teorías sobre literatura, medios de comunicación y manejo de la lengua, pero no hay referencias a las teorías sobre el desarrollo o el aprendizaje de la escritura. 
lograrla. Posteriormente, en 1979, Flavell proclamó la metacognición como a new area of cognitive-developmental inquiry $\mathrm{y}$, a partir de entonces, las investigaciones sobre este fenómeno han dado cuerpo a un importante núcleo de teoría, especialmente en el ámbito de la psicología cognitiva. Para Flavell (1976), la metacognición consiste en el monitoreo, regulación y orquestación de los procesos cognitivos al servicio de una meta concreta. Quizás uno de los principales aportes de este investigador al estudio sobre el aprendizaje humano es la distinción entre dos tipos de procesos que se dan en cualquier tarea de aprendizaje: los cognitivos y los metacognitivos. Según Flavell (1979), las estrategias cognitivas se utilizan para realizar progresos cognitivos; las estrategias metacognitivas, en cambio, se utilizan para monitorearlos.

Flavell (1979) propone un modelo de metacognición que comprende cuatro elementos: conocimiento metacognitivo, experiencias metacognitivas, metas o tareas, y estrategias. El conocimiento metacognitivo abarca los saberes y creencias que la persona tiene sobre sus propias capacidades, sobre las diversas maneras de aprender y sobre los distintos tipos de comprensión que se pueden alcanzar. Además, involucra el conocimiento sobre la tarea, es decir, sobre la información disponible y cómo manejarla para resolver un desafío concreto, y sobre el nivel de dificultad de la meta que se quiere alcanzar. Por último, el conocimiento metacognitivo supone saber distinguir las estrategias más efectivas para lograr diferentes metas. Las experiencias metacognitivas corresponden a las experiencias conscientes, cognitivas y afectivas, que acompañan cualquier desafío intelectual. Las metas y las estrategias, por su parte, corresponden a los objetivos que se propone el individuo frente a una determinada tarea cognitiva y a las herramientas que emplea para lograrlos. Flavell ha desarrollado un cuerpo teórico, sobre todo, en torno al conocimiento metacognitivo. Otros autores han investigado con mayor profundidad otros aspectos involucrados en este proceso, como la dimensión afectiva y motivacional.

Baker \& Brown (1984) retoman el concepto de metacognición de Flavell (1976) y lo utilizan para explorar los procesos involucrados en el aprendizaje de la lectura. Para estas investigadoras, la metacognición consiste en el conocimiento y control que el niño tiene sobre su propio pensamiento y aprendizaje. Según las autoras, una diferencia que distingue a los lectores expertos de los novatos es el grado de conciencia que manifiestan los lectores con más experiencia frente a las dificultades en la lectura, y su capacidad de realizar acciones reguladoras para resolver estas dificultades. Baker \& Brown (1984) proponen que mientras más explícito y detallado es el conocimiento sobre las estrategias y reglas que facilitan una lectura efectiva, más fácilmente estas pueden ser entrenadas y aprendidas. Los experimentos realizados y revisados por estas autoras parecen indicar que involucrar a los niños en actividades que requieran hacer explícitos sus conocimientos metacognitivos contribuiría al aprendizaje.

El grado de conciencia y el nivel en que se explicitan los conocimientos sobre diversas tareas parecen ser elementos clave en las teorías de la metacognición o de autorregulación del aprendizaje y, por lo mismo, tienen implicancias significativas para la enseñanza y el aprendizaje de la escritura. La pregunta por el nivel de conciencia, sin embargo, ha sido objeto de debate desde antes de la psicología cognitiva y las teorías sobre metacognición. Vygotsky, por ejemplo, afirmaba que:

Varios estudios han mostrado que es precisamente durante los primeros años de la etapa escolar que las funciones intelectuales superiores, cuyos rasgos fundamentales son el conocimiento 
reflexivo y el control deliberado, pasan al primer plano del proceso de desarrollo (...). Sin embargo, el hecho establecido por Piaget no puede ser negado: aunque el escolar avanza firmemente en cuanto a conocimiento y dominio, no tiene conciencia de sus propias operaciones conceptuales. Todas las funciones mentales básicas se tornan deliberadas durante la edad escolar, salvo el intelecto (1964: 73).

La pregunta para los investigadores de los procesos metacognitivos, propuesta por Vygotsky (1964) y discutida hasta el presente, es, justamente, acerca de la capacidad de los estudiantes de hacer conscientes los procesos mentales que les permiten aprender. Las investigaciones actuales, a partir de Flavell (1976, 1979), han reunido evidencia de que mientras más explícitos sean los conocimientos sobre la manera de aprender, más efectivo resulta el aprendizaje y, por otra parte, que la instrucción favorecería esta condición.

Las Bases Curriculares recogen someramente, en cuanto al aprendizaje de la escritura, la preocupación por el conocimiento y el grado de conciencia sobre los propios procesos de aprendizaje que los estudiantes deben manejar, es decir, por el desarrollo de la metacognición. En primer lugar, en el objetivo de escritura libre, en que los estudiantes deben seleccionar el género más adecuado de acuerdo con su propósito, se percibe la necesidad de que reflexionen sobre sus decisiones de tema y formato para escribir. Sin embargo, debido a que este objetivo es, justamente, de escritura libre, pareciera que el propósito del mismo es más bien desarrollar la motivación por la escritura que la metacognición.

En segundo lugar, en el caso de los objetivos de escritura guiada, en la introducción se estipula que:

esta aproximación permite que los alumnos se familiaricen con las ventajas y los desafíos que presenta la estructura de cada género. Para lograr lo anterior, se requiere incorporar las etapas del modelado, la práctica guiada y la práctica independiente, de modo que los estudiantes aprendan a usar los recursos para mejorar su escritura (Ministerio de Educación, 2012a: 40).

Una interpretación de estos objetivos, a la luz de la noción de metacognición, supone que los estudiantes desarrollen gradualmente autonomía para evaluar el uso de las estructuras de los géneros discursivos y para adaptarlas a sus propósitos de escritura. Ahora bien, para alcanzar mayores grados de autonomía se requiere no solo que los alumnos adquieran conocimientos explícitos sobre los géneros, sino que deben desarrollar también conciencia sobre sus propósitos de escritura, sobre las características de su posible lector y sobre las restricciones que impone la situación comunicativa particular a sus propósitos, tanto como ser capaces de orquestar todos estos dominios de saber durante el proceso de escribir. En efecto, y según las teorías aquí reseñadas, a medida que los estudiantes adquieran más conocimientos y alcancen mayores grados de conciencia, tendrán más flexibilidad para manejar las estructuras textuales y orientar sus decisiones al cumplimiento de sus objetivos, mientras escriben. Cuando los alumnos tienen altos niveles de metacognición, la escritura deja de ser una actividad mecánica en que rellenan una hoja o un esquema sin tener claridad de sus propósitos y de cómo alcanzarlos. Por lo tanto, según las nociones revisadas, favorecer la metacognición es un objetivo que debe acompañar al aprendizaje de la estructura de los géneros, y que sin embargo, no está explicitado en el currículum. Se estima que, tal como están formulados los objetivos de escritura guiada, se corre el riesgo de que se trasladen a la sala de clases como la enseñanza descontextualizada de estructuras textuales que los estudiantes terminan memorizando o replicando 
mecánicamente, en lugar de usar de manera flexible e intencionada. Por ejemplo, una explicación sobre la necesidad de desarrollar la autorregulación por la vía de monitorear la aplicación de las partes y las convenciones del género que se ha optado por utilizar, tendría el potencial de evitar una práctica pedagógica descriptiva.

Por su parte, los objetivos referidos al proceso de escritura plantean ciertas exigencias que, al igual que en los objetivos de escritura guiada, aluden implícitamente a la necesidad de promover la metacognición de los estudiantes. Debido a que se espera que estos tomen decisiones respecto de la información, la organización y el vocabulario de los textos que escriben para "satisfacer un propósito y transmitir sus ideas con claridad", es posible afirmar que, de acuerdo con las propuestas de Flavell $(1976,1979)$ y Baker \& Brown (1984), se requiere que desarrollen un cierto grado de metacognición para lograr estos objetivos de aprendizaje. Son especialmente relevantes en este contexto aquellos conocimientos referidos a los requerimientos y objetivos de la tarea -según propone Flavell (1979)-, que se construyen a través de la experiencia, pero pueden también ser explicitados en la enseñanza (por ejemplo, en experiencias metacognitivas antes, durante y después de la escritura). En este caso, por ejemplo: cuál es el propósito que se espera lograr, qué género discursivo pudiera cumplir ese propósito, cuáles son las características de ese género, qué pasos se seguirán y cómo se administrarán el tiempo y los recursos para ir logrando metas específicas orientadas al cumplimiento del propósito (buscar información, ordenarla de manera lógica, llamar la atención del lector, asegurar la coherencia del texto, entre otras metas específicas que orientan el proceso).

En breve, se propone que los objetivos curriculares referidos al proceso de escritura deberían considerar la noción de metacognición, de manera de establecer con claridad determinados ámbitos de conocimiento que los estudiantes deberían adquirir de manera explícita (lingüísticos, discursivos, de la tarea de escritura, de sí mismos como escritores) y de qué manera estos se hacen funcionales para una escritura autónoma y autorregulada. La noción de metacognición podría articular funcionalmente los conocimientos y las conductas de proceso, para promover una didáctica con enfoque comunicativo y orientada hacia niveles graduales de autorregulación en la producción escrita.

\subsection{CONCIENCIA METALINGÜÍSTICA}

$\mathrm{Al}$ igual que la metacognición, la conciencia metalingüística es un concepto que no aparece mencionado explícitamente en las Bases Curriculares de Lenguaje, ni como teoría sobre el aprendizaje de las habilidades comunicativas, en la introducción, ni como objetivo de aprendizaje. Sin embargo, del mismo modo que en el caso anterior, varios objetivos de la propuesta (especialmente los de la línea de manejo de la lengua) solo pueden ser abordados en coherencia con el enfoque comunicativo -declarado en la introducción- si es que se implementan como parte del desarrollo de la conciencia metalingüística de los estudiantes. Luego de una breve revisión sobre el desarrollo del concepto de conciencia metalingüística en la literatura, se analizará cómo informa la propuesta curricular y cómo dialoga con los objetivos de aprendizaje.

En el ámbito del desarrollo de las habilidades comunicativas varios investigadores han estudiado cómo influye el conocimiento y control sobre el lenguaje en las tareas de lectura y producción de textos (Galambos \& Goldin-Meadow, 1990; Bialystok, 1992; Karmiloff-Smith, Grant, Jones, Sims \& Cuckle, 1996; Camps y Milian, 2000). Es decir, 
las teorías acerca de la metacognición han dado pie a investigaciones sobre el conocimiento y el dominio específicos que se requieren para regular las actividades lingüísticas, en otras palabras, la conciencia metalingüística.

El concepto conciencia metalingüística destaca el rol que juega la conciencia en el proceso de aprendizaje de la lengua. Los autores recién mencionados concuerdan en que uno de los elementos que distingue a un lector o escritor novato de uno experto es el mayor grado de conciencia que este último posee sobre la actividad lingüística, es decir, sobre sus conocimientos de la lengua y cómo los utiliza en una situación particular. Esta distinción es relevante porque admite que los lectores o escritores novatos poseen diversos conocimientos sobre la lengua, la lectura o la escritura, pero especifica que gran parte de este conocimiento es implícito, es decir, no está disponible para ser expresado verbalmente o controlado a voluntad. Ahora bien, los autores también concuerdan en que no se puede hacer una diferenciación tajante entre individuos totalmente inconscientes y aquellos que poseen total conciencia sobre sus procesos cognitivos. Por el contrario, lo que parece haber, como señalan Galambos \& Goldin-Meadow (1990), es un continuиm entre diferentes niveles de conciencia, donde el mayor grado implica ser capaz de verbalizar los juicios metalingüísticos. Para estas autoras, la conciencia metalingüística consiste en la capacidad de manipular y reflexionar acerca de distintas propiedades del lenguaje (semántica, sintáctica, fonética, pragmática).

Bialystok (1992), si bien no utiliza el término conciencia metalingüística, se refiere al mismo fenómeno que describen Galambos \& Goldin-Meadow (1990), es decir, la capacidad de reflexionar sobre la lengua, y señala que el dominio lingüístico comprende dos componentes de procesamiento del lenguaje: análisis del conocimiento lingüístico y control de la atención. El análisis del conocimiento lingüístico es el proceso que permite reorganizar las representaciones mentales implícitas para volverlas explícitas y, por ende, manipulables. El control de la atención, por su parte, permite enfocarla en diferentes aspectos para resolver un problema lingüístico. Según la autora, ambos procesos se usan para resolver problemas con distinto nivel de dificultad. Lo que marca la diferencia en el grado de éxito de resolución de estos problemas es el nivel de dominio del análisis y la capacidad de control. En otras palabras, en la misma línea que Vygotsky (1964), Flavell (1976, 1979), y Baker \& Brown (1984), Bialystok (1992), reafirma que mientras más explícitos sean los conocimientos que posea el estudiante y mayor el control para atender diversos aspectos de un problema (lingüístico), mayores serán las posibilidades de éxito para alcanzar la meta.

La didáctica de la escritura se ha nutrido en gran medida de las teorías sobre conciencia metalingüística, aun cuando no siempre aborden estos términos explícitamente. Por ejemplo, Camps, Guasch, Milian y Ribas (2000) utilizan el término actividad metalingüística con un sentido similar al de conciencia metalingüística y señalan que esta "se realiza en diferentes niveles de explicitación, desde la simple manipulación del lenguaje hasta la verbalización explícita de la actividad reflexiva que recurre a una terminología metalingüística" (2000: 136). Para estas autoras, el progreso en la actividad metalingüística exige conocer conceptos específicos de las ciencias del lenguaje que permitan a los estudiantes referirse y reflexionar acerca de las producciones lingüísticas. Además de estos conceptos, el aprendizaje de la escritura requiere desarrollar conocimientos relativos a la situación comunicativa específica en que se realizará la tarea de escritura, acerca del proceso de escritura y sobre el género discursivo: "los alumnos deben ser conscientes 
de las características que definen el género discursivo con el que deben enfrentarse en el aprendizaje de la lengua (rasgos discursivos, textuales, lingüísticos)" (Camps et al., 2000: 139). Según las investigadoras, la adquisición y manejo del conocimiento lingüístico, y el desarrollo de actividades metalingüísticas en el aula posibilitan mayores grados de control y autonomía sobre la producción escrita y, por lo tanto, favorecen "las posibilidades de mejorar las habilidades orales y escritas de los alumnos, especialmente en géneros vinculados a temáticas académicas y situaciones formales" (Camps et al., 2000: 136).

Si bien la noción de conciencia metalingüística no se explicita, es posible afirmar que las Bases Curriculares recogen implícitamente los aportes de esta teoría. La evidencia más clara es la afirmación que se hace en la introducción respecto de que "el enfoque comunicativo que sustenta esta propuesta curricular considera que los conocimientos gramaticales son una herramienta para reflexionar sobre el lenguaje y sus posibilidades en contextos concretos de comunicación" (Ministerio de Educación, 2012a: 40). De acuerdo con esto, la línea de objetivos de manejo de la lengua, específicamente, aquellos referidos al aprendizaje de la gramática, estarían orientados a desarrollar la conciencia metalingüística de los estudiantes. Esta declaración, sin embargo, se refleja de manera parcial en los objetivos de aprendizaje, pues si bien se explicita la relación propuesta por los autores aquí reseñados entre conocimientos y desempeño en la actividad lingüística, la reflexión como oportunidad de aprendizaje no se desarrolla y la propuesta de aprendizaje es restringida.

Por ejemplo, en $3^{\circ}$ básico se estipula que los estudiantes aprendan la función de los pronombres y los usen "para ampliar las posibilidades de referirse a un sustantivo en sus producciones" (OA 21, 3 básico). Este objetivo es restringido a la luz de la teoría aquí revisada, por varias razones. En primer lugar, no explicita los ámbitos de reflexión sobre la función de los pronombres (por ejemplo, evitar la redundancia, mantener el sujeto lógico y en ese sentido guiar al lector; ser sujeto gramatical de oraciones). En segundo lugar, no se explicita cómo ni en qué circunstancias los estudiantes realizan esta reflexión (durante la lectura, durante la escritura, en presencia de ambigüedad causada por pronombres mal utilizados, etcétera). En tercer lugar, la funcionalidad de este conocimiento se limita a ampliar la variedad de formas de expresión ("ampliar las posibilidades de referirse a un sustantivo..."), omitiendo cuestiones clave del desempeño comunicativo, como el aporte de los pronombres al logro de la cohesión textual.

De manera similar, respecto de los verbos en $4^{\circ}$ básico se prescribe que comprendan la función de los verbos y que los usen manteniendo la concordancia con el sujeto; en $5^{\circ}$ básico se espera que conjuguen correctamente los verbos regulares y, en $6^{\circ}$ básico, que utilicen correctamente algunos participios irregulares de uso común. A la luz de la noción de conciencia metalingüística, estos objetivos son restringidos pues no prescriben que los estudiantes aprendan a reflexionar sobre la lengua y porque limitan el potencial ámbito de conocimiento de la misma. Respecto de esto último, los objetivos referidos a los verbos se centran en la normativa (concordancia, conjugación correcta), dejando de lado implicancias semánticas de los usos verbales (por ejemplo, efectos de significado al utilizar distintos tiempos y modos verbales), así como pragmáticas (por ejemplo, selección de modo indicativo para transmitir certezas al lector). Estas restricciones son, además, incoherentes con lo que se señala en la introducción del documento curricular, respecto de que en las tareas de escritura, los estudiantes deben escribir "para algo y para alguien" (Ministerio de Educación, 2012a: 40). En otras palabras, los objetivos son 
también restringidos en la manera como presentan el desempeño gramatical, pues se pierde de vista el principio sobre el aprendizaje de la escritura que recogen Camps et al. (2000), según el cual "quien escribe debe poseer una intención, un lector y un contexto en el que situará el texto a elaborar" (138). Debido a sus limitaciones, estos objetivos podrían traducirse en la sala de clases en el aprendizaje de memoria de definiciones de clases de palabras, o de conjugaciones verbales, o en ejercicios mecánicos de reemplazo de sustantivos por pronombres, en desmedro del propósito de desarrollar el conocimiento y la conciencia metalingüística para controlar las producciones escritas y autorregular las actividades de escritura en el marco de un enfoque comunicativo.

Respecto de los demás objetivos, es posible pesquisar el grado en el que promueven la conciencia metalingüística, según si asocian los distintos contenidos lingüísticos contemplados con las tareas de verbalización, reflexión y control que se derivan de la teoría aquí revisada. En el caso de los objetivos referidos al proceso de producción escrita, estos se redactan de manera que el énfasis está puesto en el control de la estructura y componentes de un texto (por ejemplo, "organizan las ideas en párrafos separados con punto aparte"), pero no se explicita la necesidad de reflexionar sobre dichos componentes o de hablar de ellos como requisito previo para poder controlar su efectividad comunicativa. Otro tanto es cierto sobre los objetivos de escritura guiada, que detallan las características de los textos que deben producir los estudiantes (por ejemplo, "escribir artículos informativos para comunicar información sobre un tema, presentando el tema en una oración, desarrollando una idea central por párrafo, utilizando sus propias palabras"), de modo que es posible inferir la necesidad de un dominio de cada uno de estos elementos para alcanzar la conducta final. Sin embargo, no hay en la redacción indicaciones de conductas intermedias, como el análisis o reflexión sobre dichas características, sus funciones y efectos de sentido, que pudieran encaminar a los estudiantes hacia el control.

En resumen, del análisis del documento curricular se puede inferir una intención de promover en los estudiantes el control de los elementos lingüísticos, orientado a la eficacia de la comunicación; sin embargo, sería necesario explicitar aprendizajes clave que se encaminan hacia dicho control, en atención a lo que dice la teoría sobre este punto. Específicamente, el análisis o reflexión acerca de los distintos componentes lingüísticos y discursivos de los textos (sus formas, funciones y efectos de sentido), así como la verbalización de los mismos, son requisitos para que los usuarios de la lengua la manipulen conscientemente.

\section{MODELOS DEL PROCESO DE ESCRITURA}

\subsection{REVISIÓN BIBLIOGRÁFICA}

Todo el eje de escritura de las Bases Curriculares se plantea desde una lógica que concibe esta actividad como un proceso cognitivo complejo. El hecho de que en cada curso se expliciten aprendizajes específicamente referidos a dicho proceso es una muestra de que se le da importancia y se prescribe no solo el producto que entrega el alumno, sino también la manera en que lo desarrolla. Esta propuesta es coherente con las teorías actualizadas, desde las que se han elaborado diversos modelos para explicar cómo funcionan los procesos cognitivos que intervienen en las actividades de escritura. A continuación se describen 
cinco modelos de escritura para luego identificar los elementos de estos modelos que están presentes y ausentes en las Bases Curriculares. Los cuatro primeros, elaborados en el marco de la psicología cognitiva, fueron seleccionados, ya que en ellos se basa gran parte de los aportes de la didáctica de la escritura. Dichos modelos son: Flower \& Hayes (1981); dos modelos de Bereiter \& Scardamalia (1987, 1992), decir el conocimiento y transformar el conocimiento; y la reformulación de Hayes (2000) a su modelo anterior. Además, se seleccionó el modelo sociocognitivo, pragmalingüístico y didáctico del grupo DIDACTEXT (2003), debido a que corresponde a una teoría hispanoamericana sobre el proceso de escritura, que además de considerar los elementos cognitivos presentes en los otros modelos, aborda el vacío de la dimensión sociocultural, que no es posible rastrear en los cuatro modelos de la psicología cognitiva.

Flower \& Hayes (1981) propusieron un modelo que criticaba y se oponía a la concepción de la escritura como una actividad que consta de varias etapas lineales. Según ellos, la escritura es una actividad compleja en la que el escritor está constantemente revisando lo que ha escrito, pensando en lo que dirá a continuación, buscando nueva información y reorientando todo el proceso para adecuarse a la meta propuesta. Incluso, señalan, las metas originales son modificadas por lo que se ha escrito y, por lo tanto, el modelo por etapas no sirve para explicar lo que ocurre realmente en la mente del escritor.

En el modelo propuesto por Flower \& Hayes se identifican varios procesos distintivos y jerárquicos que están fuertemente imbricados: la planificación, que consiste en la generación y organización del contenido orientado a metas; la traducción ${ }^{3}$, que consiste en poner el contenido que se desea comunicar por escrito; y la comprobación, que abarca la revisión y evaluación del contenido. Estos procesos son recursivos, es decir, cada uno puede estar incrustado en otra instancia mayor en los distintos momentos de la escritura; por ejemplo, se puede evaluar la idea que se acaba de generar para cumplir una meta concreta, antes de traducirla al papel. La orquestación de estos procesos estaría regulada, según los autores, por un monitor que determina, estratégicamente, cuándo pasar de uno a otro.

Además de describir los procesos involucrados en la escritura, el modelo supone que dicha actividad está constreñida por el contexto de la tarea (task environment), que involucra las limitaciones que ponen al autor el problema retórico y el texto producido hasta el momento. Asimismo, todo el proceso de escritura está delimitado por los conocimientos del autor sobre el tema, la audiencia y los planes de escritura, que están almacenados en su memoria a largo plazo. Este modelo permite, por lo tanto, visibilizar la complejidad del proceso, que requiere coordinar varias habilidades y conocimientos que concurren simultáneamente en las actividades de escritura.

Hayes (2000), en su reformulación del modelo, incluye, además de los factores recién mencionados, la memoria operativa como factor central en el funcionamiento de todo el proceso. De acuerdo con Hayes, la memoria operativa es el recurso cognitivo que permite a los escritores enfocar su atención en ciertos elementos y mantener en un nivel automático aquellos procesos que no suponen problemas. Aquello que se vuelve consciente para el escritor sería, por lo tanto, lo que necesita de su atención, ya sea para resolver

Los autores utilizan el término traducir (translate) en vez de transcribir o escribir para destacar las peculiaridades de este proceso, en el que el escritor organiza una compleja red de conceptos para transformarla en una pieza de escritura lineal y comprensible para el lector. 
una dificultad o tomar una decisión frente a varias alternativas. Además de otorgar un rol central a la memoria operativa, en este nuevo modelo Hayes incluye la motivación y los aspectos afectivos como dimensiones que constriñen las actividades de escritura y que el anterior modelo no contemplaba.

Uno de los principales aportes de los modelos de Bereiter \& Scardamalia (1987, 1992) es que permiten explicar las maneras diferentes en que operan escritores expertos y novatos. Estos autores amplían la teoría de Flower \& Hayes (1981), que estipula que las diferencias entre novatos y expertos se relacionan en gran medida con su manejo de las metas de escritura, y proponen que "la principal diferencia existente entre la composición madura e inmadura está en la manera de introducir el conocimiento y en lo que le sucede a ese conocimiento a lo largo del proceso de composición" (Bereiter y Sacardamalia, 1992: 44). El modelo que explica la escritura propia de los escritores novatos se conoce como decir el conocimiento y expone la manera en que los escritores inmaduros aseguran la coherencia de sus textos sin necesidad de aplicar deliberadamente su conocimiento sobre el mundo y los géneros. Según este modelo, los escritores se crean una representación mental de lo que deben decir y luego localizan identificadores de tópico y de género. Los identificadores de tópico sirven como pistas para buscar en la memoria información asociada al tema de escritura. Una vez que se localiza y se escribe cierta información, esta misma sirve como nuevo identificador del tópico, que permite encontrar contenido relacionado con lo que se acaba de escribir. Así se asegura un cierto grado de coherencia, gracias a la influencia que ejerce un contenido recuperado en el que se busca a continuación. Los identificadores de género funcionan de la misma manera que los de tópico $\mathrm{y}$, en conjunto con estos, permiten generar y, a la vez, limitar el número de ideas sobre un tema hasta que estas se agoten o se acabe el espacio para escribir.

El modelo transformar el conocimiento, por su parte, permite describir los procesos que utiliza un escritor maduro para generar un texto. Este modelo contiene varias semejanzas con el de Flower \& Hayes (1981); por lo pronto, también involucra varios procesos y subprocesos que se superponen y funcionan de manera recursiva. Bereiter \& Scardamilia (1987, 1992) describen dos espacios de problemas que dan cuenta de los conocimientos y operaciones involucradas en las decisiones de un escritor experto: el espacio-problema del contenido y el espacio-problema retórico. El espacio del contenido comprende, por un lado, los estados de conocimiento del autor, es decir, las creencias que posee sobre el tema del texto y, por otro lado, supone que los escritores, al escribir, realizan hipótesis y deducciones que les permiten alterar sus creencias. El espacio-problema retórico, por su parte, abarca las representaciones sobre el texto y sus objetivos subordinados, y las operaciones que alteran el texto, los objetivos o las relaciones entre ambos. El espacio retórico se ocupa de cumplir las metas discursivas y de las relaciones entre el contenido del texto y las posibles reacciones de los lectores. Según este modelo, la recuperación de la información funcionaría básicamente de la misma manera que en el modelo anterior, sin embargo, esta debe adaptarse a las restricciones específicas originadas por el análisis del escritor del problema retórico. De esta manera, el contenido puede provocar ciertos problemas retóricos que, a su vez, producen problemas en el espacio del contenido, y así sucesivamente. Mediante este proceso, el conocimiento se va transformando, a fin de resolver los objetivos y problemas del espacio retórico.

En cuanto al modelo sociocognitivo, pragmalingüístico y didáctico del grupo DIDACTEXT (2003), basado en la reformulación de Hayes (2000), se destaca su 
incorporación de la dimensión cultural a la actividad de escritura, que los otros modelos aquí revisados no abordan explícitamente. El modelo DIDACTEXT concibe la escritura como una actividad en la que interactúan recursivamente tres dimensiones simbolizadas por círculos concéntricos: el ámbito cultural, los contextos de producción y el individuo.

El ámbito individual de este modelo de escritura recoge las mismas dimensiones interrelacionadas que la propuesta teórica de Hayes (2000): memoria, motivación-emociones y estrategias cognitivas y metacognitivas. Además de la memoria cognitiva, este modelo incorpora la memoria cultural como un factor clave en los procesos de producción escrita. El principal supuesto que subyace al concepto de memoria cultural es que la mente se construye en relación con el contexto. Esto significa que "Cada cultura posee su propia forma de simbolizar el tiempo, el espacio, la naturaleza, los sonidos, las ideas" (DIDACTEXT, 2003: 88).

El segundo círculo del modelo DIDACTEXT se refiere a los contextos de producción que delimitan las actividades de escritura. Hayes (2000) entendía este contexto como el contexto social (audiencia y colaboradores) y el medio de composición, pero estos autores amplían esta noción incorporando el contexto situacional y el contexto físico. El contexto social comprende aspectos políticos, educativos, jurídicos, laborales, económico-comerciales, familiares, las relaciones cotidianas y el ocio, que se interrelacionan de manera dinámica y difusa, es decir, no es posible percibir límites claros entre cada uno de estos aspectos. El contexto situacional, por su parte, además de la audiencia y los colaboradores con todas sus características, incluye a los detractores del texto, al entorno geográfico y al entorno inmediato donde ocurre la actividad de escritura. Estos últimos, según los autores, son aspectos clave en cuanto afectan la cultura de cada grupo humano. Por último, el contexto físico "se refiere al lugar (espacio físico) y al medio de composición utilizado para la ejecución de la tarea de escritura" (DIDACTEXT, 2003: 84). Todos estos elementos dan forma y son alterados por los artefactos culturales de la sociedad y, en este sentido, constituyen parte de la memoria cultural que las personas incorporan a su memoria individual como conocimientos, creencias y prejuicios.

Finalmente, el primer círculo, que incluye a los otros dos, es el de la cultura. Dentro de este ámbito, los autores desarrollan el concepto de género discursivo. Los géneros discursivos son la forma típica y relativamente estable en que, según las diversas esferas de la praxis humana, se estructuran los enunciados. Son, siguiendo a Bajtin (1979, Cit. en DIDACTEXT, 2003), registros sociales en que se plasman los discursos, delimitados por el contexto social, la forma de enunciación y el tema. De esta teoría se desprende que los géneros discursivos permitirían enmarcar las actividades de escritura en formas concretas de enunciación que dan cuenta del ámbito cultural en que se realiza el discurso. De acuerdo con esto, desarrollar la conciencia metalingüística, tomando en consideración los aportes del modelo DIDACTEXT y en la línea de la propuesta didáctica de Camps et al. (2000), supondría incluir el análisis de las características y funciones de los géneros discursivos, de manera de traer al foco de atención el ámbito de la cultura que constriñe las producciones escritas.

\subsection{ANÁLISIS DE LOS OBJETIVOS DE APRENDIZAJE A LA LUZ DE LOS MODELOS REVISADOS}

Un análisis de los objetivos de aprendizaje a la luz de los modelos de Bereiter \& Scardamalia (1987, 1992), devela, en primer lugar, un vacío respecto de orientaciones específicas para que los escritores novatos puedan hacerse cargo de los problemas que 
les plantea la articulación entre el espacio del contenido y el espacio retórico. Como proponen los autores, el modelo experto se caracteriza por ser capaz no solo de "decir" contenidos en el texto, sino también de "transformarlos", entendido esto último como una oportunidad para desarrollar más y aprender más sobre estos contenidos, en la medida en que se vuelve críticamente sobre ellos para relacionarlos y estructurarlos por escrito. La ausencia de orientaciones en los objetivos y en la introducción respecto de la transformación de los conocimientos puede conducir a que, tal como temen los autores, el proceso de escritura sea aplicado por los estudiantes para realizar correcciones cosméticas a sus textos, en lugar de reestructuraciones profundas relativas a los significados y a grandes porciones del texto. En este sentido, sería conveniente, por una parte, explicitar en algún objetivo (por ejemplo, en el de escritura de artículos informativos) que los estudiantes escriben para reformular un conocimiento, hacer una síntesis, revisar sus ideas, entre otros. En la introducción, por otra parte, podrían entregarse algunas claves de cómo orientar la escritura según el modelo de transformación del conocimiento; por ejemplo, a través del uso de mapas conceptuales u otros esquemas que impongan a los estudiantes preguntarse sobre sus conocimientos y las relaciones entre ellos, antes de escribir. De este modo, el currículum se haría cargo de la escritura como herramienta de aprendizaje.

Respecto del dinamismo de los momentos del proceso de escritura, que describen Flower \& Hayes (1981) y Hayes (2000) en contraposición con los modelos de etapas, las Bases Curriculares aquí revisadas realizan una opción en general coherente. En efecto, separan la etapa de la planificación y agrupan escribir, revisar y editar, en lo que parece una indicación de que estos tres últimos momentos pueden ocurrir de manera simultánea y recursiva. Así también, en $5^{\circ}$ básico se explicita la relectura, destacada en el modelo de Hayes (2000), propuesta que podría ser enriquecida para acercarse aún más a la visión del autor. Vista la propuesta del autor de que leer el propio texto durante la escritura es un acto de interpretación orientado a mejorar el texto, sería deseable explicitar conductas específicas asociadas a mejoras específicas; por ejemplo, releer el párrafo recién escrito para determinar su relación con el que sigue, releer el texto completo antes de revisar el título o el párrafo introductorio, o antes de redactar la conclusión.

Otra cuestión que aparece suficientemente abordada en la propuesta es la dimensión afectiva y motivacional explicitada por Hayes (2000). En efecto, el objetivo de escritura libre, que constituye una novedad respecto de currículos anteriores, supone una oportunidad claramente definida para promover la motivación de los estudiantes y abrir un espacio para que desarrollen, por medio de la escritura, sus intereses personales y autonomía para tomar decisiones. Se estima que ambos elementos pueden favorecer una disposición positiva de los estudiantes hacia las actividades de escritura, en particular hacia aquellas más estructuradas contenidas en los demás objetivos.

Mientras que la filiación a un enfoque de proceso y a la motivación por escribir se delinea con claridad en las Bases, una dimensión mucho más oscura lo constituye el ámbito social y cultural. Si bien las Bases introducen el trabajo con géneros discursivos propios de la vida cotidiana y escolar de los estudiantes (por ejemplo, en la escritura de narraciones y textos no literarios se proponen ejemplos de géneros como anécdotas, cuentos, poemas, diarios de vida, blogs, cartas, instrucciones, afiches y, además, hay un objetivo específicamente dedicado a la escritura de artículos informativos), no se especifica la articulación que debe haber entre estos géneros y el contexto sociocultural de los mismos, como propone el modelo (DIDACTEX, 2003). En los objetivos de narraciones 
y de artículos informativos, los textos se describen en términos de sus estructuras, pero no se vinculan con el contexto en el que circulan dichos géneros, ni se especifica la relación que se establece, según el género discursivo y el contexto, con el interlocutor. Esta relación es relevante porque influye directamente en la elección de un género sobre otro, en el tipo de lenguaje (directo o indirecto), en la formalidad, en el nivel de densidad de la información, en la selección léxica, entre otras decisiones relevantes orientadas a la contextualización de los textos producidos. En efecto, la noción de género discursivo involucra conceptualizar el acto de escribir como una práctica definida por la sociedad, cuyas convenciones deben ser observadas en el marco de la interacción con otros por escrito. De esta manera, incorporar el contexto y la relación con el interlocutor permitiría abordar los géneros discursivos en su real complejidad y dinamismo; de otra forma, se corre el riesgo de que se enseñen como estructuras estáticas y descontextualizadas. Para que los estudiantes desarrollen flexibilidad y aprovechen las posibilidades que ofrecen los géneros, como proponen las Bases, sería necesario que fueran conscientes de cómo sus particulares estructuras y recursos discursivos surgen en ámbitos sociales determinados (académico, escolar, público, cotidiano, etcétera) y cumplen funciones determinadas.

Es interesante que los objetivos referidos a la escritura, revisión y edición de los textos solo incorporen la dimensión social en términos del registro de habla. Por ejemplo, en el objetivo de $3^{\circ}$ básico se señala que los estudiantes "adecuan el registro al propósito del texto y al destinatario". El término registro ha sido ampliamente utilizado en los currículos de Lenguaje y Comunicación, como la manera de incorporar los factores situacionales y culturales que intervienen en las actividades de escritura. Sin embargo, este concepto es muy limitado porque no incluye todas las dimensiones discursivas que hay que tomar en cuenta para incorporar el ámbito sociocultural: el género discursivo particular y sus convenciones características como la complejidad sintáctica, los marcadores discursivos y la densidad de la información; el tipo de información seleccionada, el vocabulario, el lenguaje directo o indirecto y la presentación gráfica de la información, todas estas nociones que varían según la situación (por ejemplo, el momento histórico, el ámbito de producción), el propósito y el destinatario del texto (más o menos cercano, más o menos informado).

Debido a lo anterior, en el ánimo de completar una dimensión clave de la producción escrita y de guardar coherencia con la inclusión de géneros discursivos en las Bases, sería conveniente especificar más elementos lingüísticos y discursivos asociados a esta noción, así como las prácticas sociales que los rigen. Por ejemplo, respecto de la escritura de artículos informativos, en lugar de presentar únicamente la estructura del género, sería deseable discutir con los estudiantes las particulares situaciones en las que este se escribe, quiénes lo escriben, por qué razones y con qué propósitos, y cómo la estructura y los recursos discursivos característicos del género contribuyen a realizar los señalados propósitos. En otras palabras, se sugiere no focalizar la enseñanza en elementos estructurales, sino más bien dar oportunidad para una reflexión metalingüística sobre la relación entre los recursos lingüístico/discursivos y la esfera social y cultural que constriñe la selección durante la escritura: el género discursivo, la formalidad de la situación (en relación con el interlocutor), los conocimientos que maneja el destinatario del texto, los giros idiomáticos y el lenguaje figurado permitidos en el espacio de circulación del texto, entre otros. De este modo, los estudiantes, con la ayuda de guía adecuada y de pautas específicas, aprenderían a tomar decisiones más conscientes en relación con la 
dimensión sociocultural y, así, podrían orientar mejor el proceso de escritura hacia el logro de sus propósitos.

\section{CONCLUSIONES}

En términos generales, las nuevas Bases Curriculares incorporan de manera clara algunos aportes de la teoría actualizada sobre escritura, aunque también presentan algunos vacíos al respecto. En cuanto a las teorías sobre metacognición y conciencia metalingüística revisadas en este trabajo, se estima que estas informan implícitamente el currículum; es decir, al ser analizados, tanto la introducción como los objetivos resultan coherentes con estas nociones. Sin embargo, se estima que para promover una didáctica de la producción escrita basada en dichas nociones sería deseable incluirlas de manera explícita en los objetivos de aprendizaje. En efecto, para formar escritores gradualmente más autónomos y autorregulados, que puedan valerse de la escritura como herramienta de aprendizaje y de comunicación, como propone el currículum, es necesaria una didáctica que, respecto de la producción escrita, desarrolle conocimientos explícitos, ofrezca oportunidades de reflexión y orientaciones para el monitoreo y la regulación. En este sentido, una dimensión especialmente débil es la propuesta de reflexión sobre los componentes gramaticales, pues, si bien se declara, no se incluyen indicaciones sobre cómo realizar esta reflexión o en qué momentos, a la vez que se ofrece una propuesta restringida sobre qué aspectos gramaticales deberían ser objeto de reflexión y cómo ello se asociaría con el desempeño escrito.

Al analizar el documento a la luz de distintos modelos del proceso de escritura, es evidente un foco claro en el proceso de escritura como una actividad compleja, compuesta de momentos que ocurren simultánea y recursivamente, y que deben ser orquestados por el escritor. Se presenta también con claridad la necesidad de que los escritores aborden esta tarea con motivación y una actitud positiva y orientada al descubrimiento de una voz propia y una actitud autónoma. Mucho más débil es la relación entre el proceso de escritura y la posibilidad de aprender. En efecto, las dimensiones de construcción y transformación del conocimiento durante la escritura, que sí se explicitan en la introducción a las Bases, no aparecen representadas en los objetivos de aprendizaje.

Otro ámbito que merece ser destacado, por su contraste con los modelos revisados, es la dimensión social y cultural de la producción escrita. Como se detalla en este trabajo, en el documento se incluye la noción de género discursivo, pero su descripción se limita a la estructura de la información y al registro de habla, dejando fuera las diversas convenciones discursivas asociadas a cada género, que surgen y son requeridas para participar en los distintos ámbitos comunicativos. Se estima que esta debilidad podría perpetuar en las aulas un tipo de enseñanza descriptiva y disociada de la comunicación contextualizada.

Finalmente, se incluye en este trabajo una breve discusión respecto de las limitaciones que impone el propósito del currículum y la manera en que se frasean sus objetivos. Específicamente, se argumenta que centrar el documento en los resultados de aprendizaje, en lugar de ampliar su rol hacia posibles orientaciones didácticas, deja en manos de la formación inicial y en servicio un trabajo arduo de análisis y de actualización teórica y práctica que necesariamente ocurre con posterioridad a la publicación e implementación 
del currículum. Sin eludir este proceso estrictamente necesario en la lógica de perfeccionamiento continuo propia de la profesión docente, se sugiere que los documentos curriculares puedan orientar esta tarea explicitando aún más las bases teóricas en las que se sustentan, así como, en la medida de lo posible, las conductas intermedias que pueden realizar los estudiantes para alcanzar los aprendizajes prescritos.

\section{REFERENCIAS BIBLIOGRÁFICAS}

Baker, L. \& Brown, A. L. (1984). Metacognitive skills in reading. In P. D. Pearson (Ed.), Handbook of reading research (pp. 353-387). New York: Longman.

Bereiter, C. y Scardamalia, M. (1992). Dos modelos explicativos de los procesos de composición escrita. Infancia y Aprendizaje, (58), 43-64. (1987). The psychology of written composition. New York: Routledge.

Bialystok, E. (1992). Attentional control in children's metalinguistic performance and measures of field independence. Developmental Psychology, vol. 28 (4), 654-664.

Camps, A. y Milian, M. (2000). El papel de la actividad metalingüística en el aprendizaje de la escritura. Rosario: Homo Sapiens.

Guasch, O., Milian, M. y Ribas, T. (2000). Actividad metalingüística: La relación entre escritura y aprendizaje de la escritura. En A. Camps y M. Milian (Eds.), El papel de la actividad metalingüística en el aprendizaje de la escritura (pp. 135-162). Rosario: Homo Sapiens.

DIDACTEXT (2003). Modelo sociocognitivo, pragmalingüístico y didáctico para la producción de textos escritos. Didáctica (Lengua y Literatura), vol. 15, 77-104. Recuperado el 1 de abril de 2013 desde http://www.didactext.net/acciones/publicacion.pdf

Flavell, J. H. (1979). Metacognition and cognitive monitoring: A new area of cognitive-developmental inquiry. American Psychologist, vol. 34 (10), 906-911.

(1976). Metacognitive aspects of problem solving. In L. B. Resnick (Ed.), The nature of intelligence (pp. 231-235). Hillsdale, NJ: Erlbaum.

Flower, L. \& Hayes, J. (1981). A cognitive process theory of writing. College Composition and Communication, vol. 32 (4), 365-387.

Galambos, S. \& Goldin-Meadow, S. (1990). The effects of learning two languages on levels of metalinguistic awareness. Cognition, vol. 34 (1), 1-56.

Hayes, J. (2000). A new framework for understanding cognition and affect in writing. In R. Indrisano \& J. Squire (Eds.), Perspectives on writing: Research, theory, and practice (pp. 6-44). Newark, DE: International Reading Association.

Karmiloff-Smith, A., Grant, J., Jones, M.-C., Sims, K. \& Cuckle, P. (1996). Rethinking metalinguistic awareness: Representing and accessing what counts as a word. Cognition, vol. 58 (2), 197-219.

Ministerio de Educación (2012a). Bases curriculares de la educación básica. Lenguaje y comunicación. Recuperado el 14 de septiembre desde http://www.mineduc.cl/index5_int. php?id_portal=47yid_contenido $=17116$ yid_seccion $=3264 \mathrm{yc}=1$

(2012b). Progresión de objetivos de aprendizaje: Lenguaje y comunicación $1^{\circ}$ a

$6^{\circ}$ básico. Recuperado el 14 de septiembre de 2013 desde http://www.mineduc.cl/index5_int. php?id_portal $=47 \mathrm{yid} \_$contenido $=17116 \mathrm{yid} \_$seccion $=3264 \mathrm{yc}=1$

Vygotsky, L. (1964). Pensamiento y lenguaje: Teoría del desarrollo cultural de las funciones psíquicas. Buenos Aires: La Pléyade. 